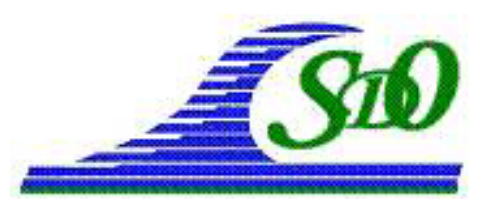

XI ìmes Journées Nationales Génie Côtier - Génie Civil

Les Sables d'Olonne, 22-25 juin 2010

DOI:10.5150/jngcgc.2010.012-K C Editions Paralia CFL

disponible en ligne - http://www.paralia.fr - available online

\title{
Caractérisation numérique et expérimentale des perturbations engendrées par la présence de tables ostréicoles
}

\author{
Youen KERVELLA ${ }^{1}$, Grégory GERMAIN ${ }^{2,3}$, Benoît GAURIER ${ }^{2,3}$, \\ Florence CAYOCCA ${ }^{1}$, Patrick LESUEUR ${ }^{4}$
}

1. IFREMER, Laboratoire PHYSED, 29280 Plouzané, France.

2. IFREMER, Serv. Hydrodynamique \& OcéanoMétéo, 62200 Boulogne/Mer, France.

3. Univ. Lille Nord de France, 59000 Lille, France.

4. Univ. Caen, Laboratoire M2C, 14000 Caen, France.

gregory.germain@ifremer.fr

\section{Résumé :}

Sur de nombreux sites ostréicoles, d'importants mouvements sédimentaires modifient les conditions d'exploitation. Les mouvements naturels sont modifiés par la présence des tables à huîtres, les phénomènes en jeu étant de deux ordres : la perturbation des courants de marée et la modification des conditions de houle.

Nous présentons dans ce document la méthodologie mise en place pour améliorer la compréhension des perturbations physiques du milieu engendrées par l'implantation de tables ostréicoles et caractériser les modifications de l'écoulement. Les résultats obtenus, tant expérimentaux que numériques, sont présentés et les développements pour une extension du modèle à l'échelle d'un parc ostréicole sont discutés.

Des essais expérimentaux réalisés à l'échelle $1 / 2$ ont notamment permis de caractériser l'impact en champ proche sur l'écoulement. Nous avons mis en évidence des zones préférentielles de dépôts de sédiments dues à l'interaction entre la couche limite se créant sur le dessous de la table et la couche limite de fond. Les contraintes de cisaillement mesurées près du fond ne sont quant à elles que très peu modifiées par la présence de la structure. Seule la contrainte de cisaillement sur l'ensemble de la colonne d'eau est amplifiée au passage des tables.

Avec l'objectif de généraliser ces travaux, un modèle numérique a été développé à partir du code de CFD Fluent, les tables étant modélisées à l'aide de milieux poreux. La comparaison des résultats numériques et expérimentaux montre une bonne concordance : de 3 à 10\% d'erreur moyenne sur l'ensemble du domaine d'étude suivant la configuration étudiée. Les choix effectués pour représenter la structure permettent d'envisager l'extension du modèle à des échelles plus importantes (plusieurs centaines de mètres) pour une structure seule ou en interaction avec d'autres placées en espace proche.

\section{Mots-clés :}

Mesures LDV - Essais expérimentaux - Simulations numériques - Contraintes de cisaillement - Hydrodynamique côtière - Table ostréicole 


\section{Introduction}

Les sites où se sont développées les activités ostréicoles sont souvent des zones abritées, telles que les baies et les bassins, pour faciliter l'exploitation des mollusques. Ces zones abritées, présentant par définition des conditions hydrodynamiques "calmes", sont des zones propices à une sédimentation vaseuse importante. Ce phénomène est amplifié du fait de la géométrie même des tables à huîtres qui constituent des obstacles artificiels pouvant perturber l'écoulement des courants de marée et la propagation des vagues, et ainsi modifier le transport sédimentaire (SORNIN, 1981). De ces deux phénomènes, il résulte des envasements locaux parfois très prononcés et menaçants pour l'activité ostréicole. Divers schémas de restructuration sont proposés localement afin de remédier à cette situation mais à l'heure actuelle, aucune étude précise des modifications hydrodynamiques inhérentes à ces installations n'a été réalisée. Il est donc impossible de prédire l'efficacité de ces plans d'aménagement à long terme. Les phénomènes sources de la modification des mouvements sédimentaires naturels sont de deux ordres : la perturbation des courants de marée et la modification des conditions de houle.

Nous présentons dans ce document la méthodologie mise en place pour améliorer la compréhension des perturbations physiques du milieu engendrées par l'implantation de tables ostréicoles et caractériser les modifications de l'écoulement. Ce travail pouvant être difficilement effectué à partir de mesures in situ (LE HIR et al., 2008), nous avons fait le choix de réaliser cette étude à partir du développement d'un modèle numérique permettant de simuler l'écoulement autour de tables ostréicoles. Ce modèle dit de moyenne échelle provient de l'extension d'une modélisation petite échelle, validée à l'aide d'essais expérimentaux réalisés en veine de circulation.

Dans la première partie de ce document, nous présentons le travail expérimental réalisé et les principaux résultats obtenus. La présentation du modèle numérique ainsi que sa validation font l'objet de la deuxième partie. L'extension du modèle petite échelle à la moyenne échelle (échelle d'un parc ostréicole) est ensuite discutée.

\section{Approche expérimentale}

Les campagnes d'essais devant être effectuées au bassin à houle et courant de l'Ifremer, nous avons choisi de représenter les structures à l'échelle $1 / 2$ (figure 1). Ce choix permet de considérer une immersion au réel de $4 \mathrm{~m}$ (valeur intermédiaire sur des estrans où elle peut varier entre 0 et $10 \mathrm{~m}$ ), tout en conservant un nombre de configurations d'essais conséquent, la veine présentant une section d'essais de $4 \mathrm{~m}$ de large, $2 \mathrm{~m}$ de haut sur une longueur utile de $18 \mathrm{~m}$. La structure ainsi réalisée (assemblage galvanisé de fer à béton de $8 \mathrm{~mm}$ de diamètre) présente une section de $0,5 \mathrm{~m}$ de large par $0,35 \mathrm{~m}$ de haut, pour une longueur finie de 7,2 m. Cette longueur ne permet pas de représenter une longueur de table réelle, mais est suffisante pour simuler les phénomènes en présence (KERVELLA et al., 2010) et constituer un cas intéressant pour la validation d'un modèle numérique. Les poches à huîtres utilisées ont un diamètre d'ouverture de maille 
de $7 \mathrm{~mm}$, une épaisseur de $6 \mathrm{~cm}$ et sont remplies de galets calibrés pour simuler la présence des huîtres à l'échelle considérée.
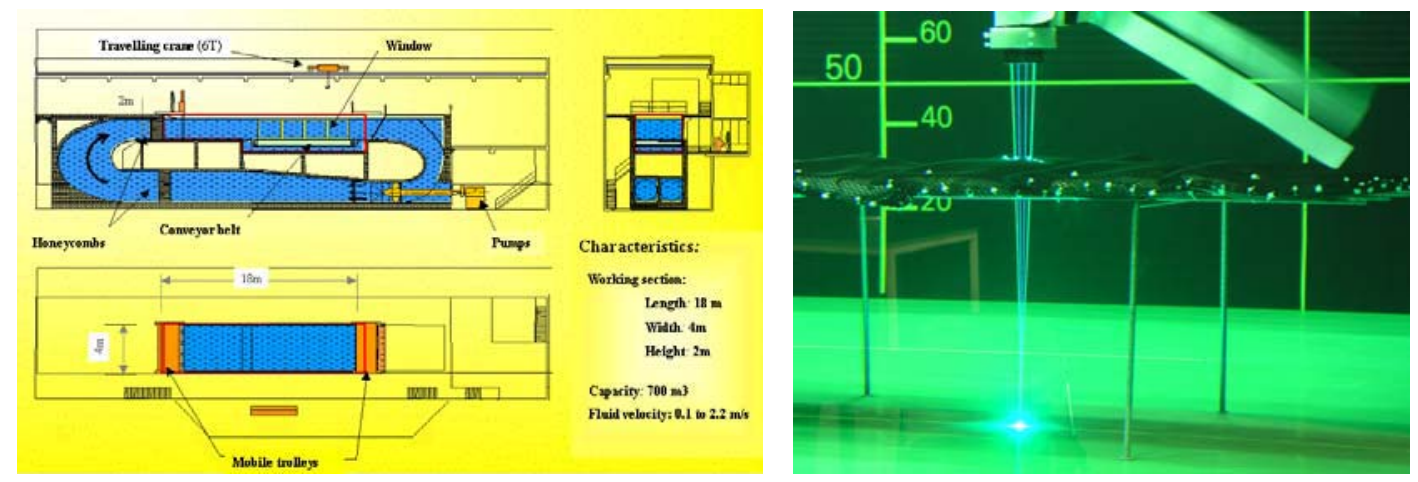

Figure 1. Caractéristiques du bassin à houle et courant de l'Ifremer et mesures LDV sous la maquette de table à huîtres.

Les conditions de courant rencontrées sur les sites d'exploitation étant assez variables, nous avons choisi de considérer trois orientations de tables par rapport à la direction de l'écoulement incident : une longueur de table de 7,2 m colinéaire au courant incident (configuration A), une longueur de table de 7,2 $\mathrm{m}$ présentant un angle de $15^{\circ}$ par rapport à la direction principale du courant (configuration $\mathrm{B}$ ) et deux longueurs de table de $3,6 \mathrm{~m}$ positionnées perpendiculairement au courant (configuration $\mathrm{C}$ ). La vitesse de courant considérée est assez élevée : $0,5 \mathrm{~m} / \mathrm{s}$ en bassin, correspondant à une vitesse réelle d'environ $0,7 \mathrm{~m} / \mathrm{s}$ (vitesse maximale rencontrée sur certains sites d'exploitation) pour faciliter l'identification des phénomènes en présence, le taux de turbulence en bassin étant relativement faible, de l'ordre de 5\% (HENDRICKS et al., 2006).

La caractérisation des perturbations engendrées par la présence de ces structures sur l'écoulement a principalement été effectuée à partir de mesures LDV (système de vélocimétrie laser). De nombreux profils verticaux ont été effectués autour et sous la structure, grâce notamment au positionnement d'ouverture sur les poches à huîtres lors des mesures sous table. La discrétisation choisie a permis d'extrapoler les mesures LDV entre deux profils pour la réalisation de cartes de vitesses (extrapolation validée à l'aide de mesures PIV, KERVELLA et al., 2010). Ainsi, l'influence des tables ostréicoles sur l'écoulement peut être étudiée, notamment à partir des champs de vitesses axiales moyennes normalisées présentées figure 2 suivant le plan de symétrie vertical de la structure pour les configurations A, B et C. Ces champs permettent de caractériser les différentes couches limites se créant sur le fond (configuration A uniquement), mais également sur et sous la table. Un déficit de vitesse de l'ordre de 30\% est atteint dans la zone de rencontre des couches limites du fond et de celle créée sous la table pour la configuration $\mathrm{A}$, alors qu'un déficit de vitesse équivalent est uniquement atteint dans le sillage immédiat à hauteur des sacs pour la configuration B (le sillage s'étend 
uniquement dans le prolongement de la structure, avec une évolution quasi nulle en épaisseur, KERVELLA et al., 2009). Les effets de masquage et d'interaction entre les tables dans la configuration $\mathrm{C}$ se traduisent par un épaississement du sillage de la table positionnée en aval de la première.

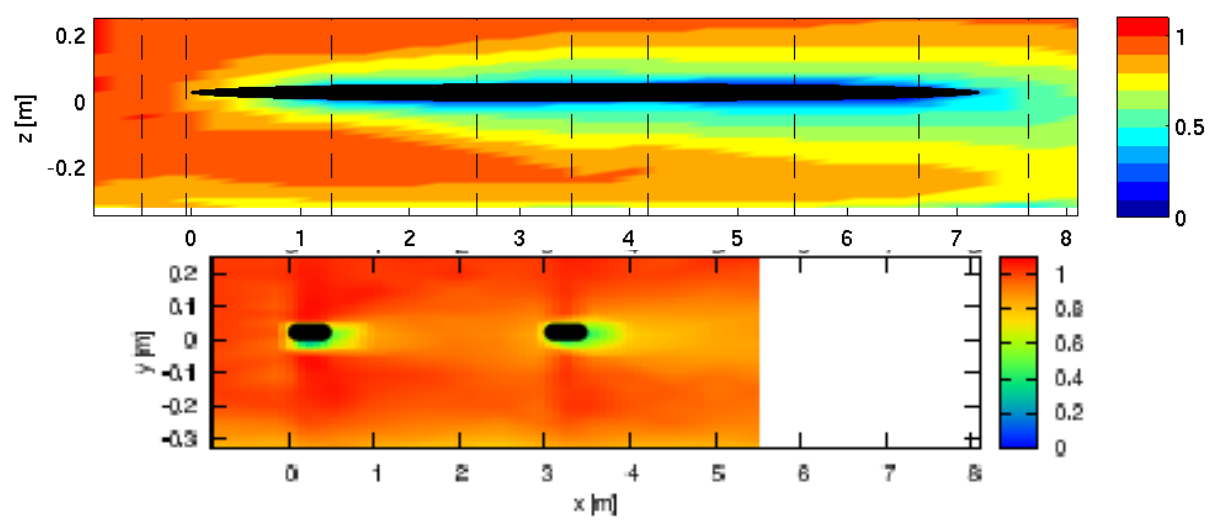

Figure 2. Champ de vitesses axiales normalisées dans le plan de symétrie vertical pour les configuration A (en haut) et $C$ (en bas).

Les mesures LDV effectuées permettent non seulement d'établir les champs de vitesses moyennes précédents, mais permettent également de déterminer les contraintes de cisaillement générées par la présence des structures dans l'écoulement. Pour cela, nous avons réalisé des mesures LDV avec des temps d'acquisitions suffisamment grands (420 secondes) pour s'assurer de la convergence de cette quantité, calculée sur les différents profils considérés : $\tau=\rho \overline{u^{\prime} w^{\prime}}$ (u et w étant respectivement la composante de vitesse axiale et verticale de l'écoulement). L'analyse de ces résultats (figure 3) montre que les contraintes de cisaillement mesurées près du fond ne sont pas modifiées par la présence de la structure. Seule la contrainte de cisaillement globale, calculée sur l'ensemble de la colonne d'eau, est amplifiée au passage des tables (tableau 1). Les valeurs obtenues dans ce cadre sont du même ordre de grandeur que les mesures ADV réalisées in situ (entre 0,05 et 0,5 Pa, VERNEY et al., 2006). L'interaction entre les deux structures de la configuration $\mathrm{C}$ se traduit par une augmentation de la proportion de l'écoulement présentant une contrainte de cisaillement élevée.

Tableau 1. Evolution de la contrainte de cisaillement le long de la structure (conf. A).

\begin{tabular}{lllll}
\hline & En amont & $1,29 \mathrm{~m}$ & $3,52 \mathrm{~m}$ & $5,50 \mathrm{~m}$ \\
\hline$\tau_{\text {bottom }}(\mathrm{Pa})$ & 0,25 & 0,25 & 0,14 & 0,14 \\
$\tau_{\text {total }}(\mathrm{Pa})$ & 0,25 & 4,45 & 2,95 & 2,58 \\
\hline
\end{tabular}

Compte tenu des restrictions expérimentales (limitation de la longueur et du nombre de structures en interaction), il est nécessaire de recourir à la simulation numérique pour 
étendre ces résultats et aboutir à une compréhension globale des phénomènes rencontrés in situ (NEPF \& GHISALBERTI, 2008). Les développements réalisés dans ce sens sont présentés dans la partie suivante, les résultats expérimentaux servant à leur validation.
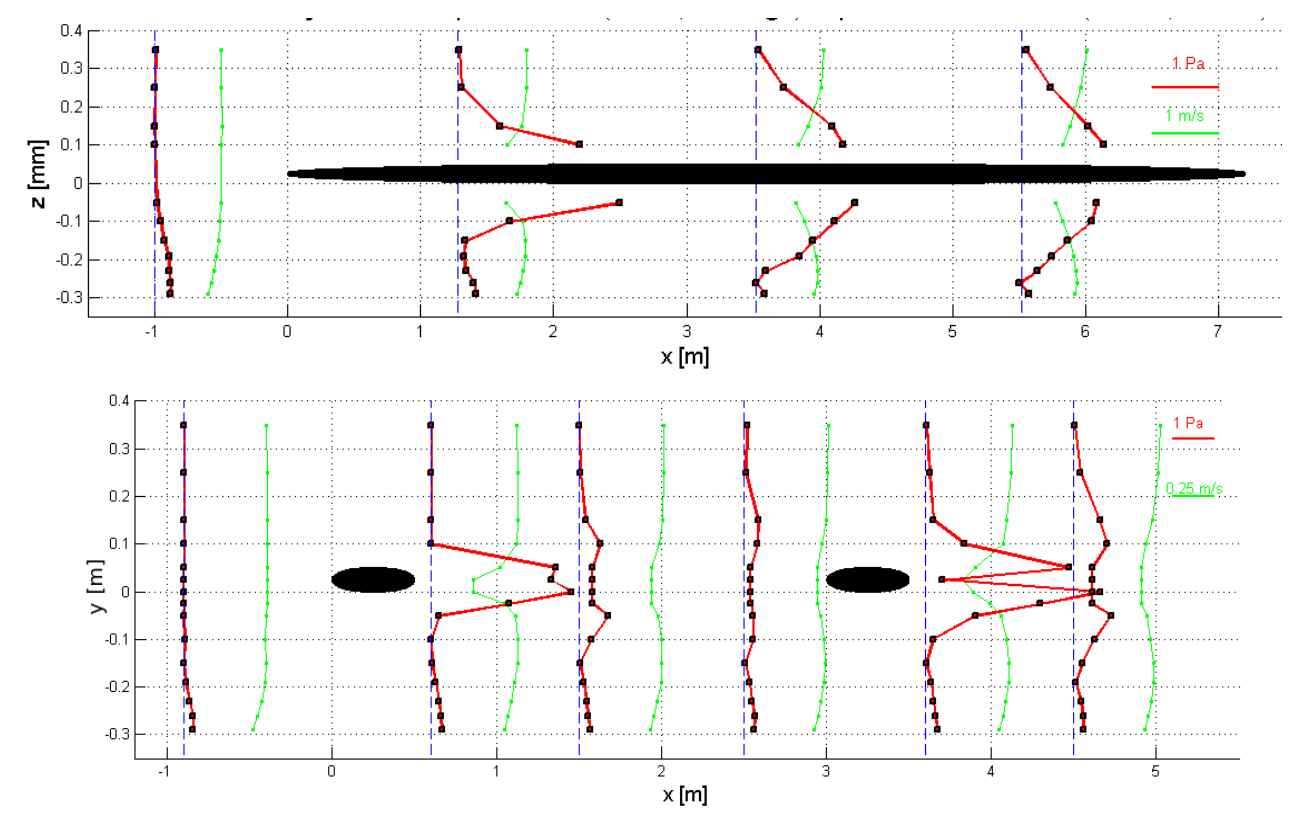

Figure 3. Evolution de la contrainte de cisaillement pour les configurations $A$ (figure du haut) et $C$ (figure du bas).

\section{Approche numérique}

Les essais expérimentaux présentés précédemment ont permis de mieux appréhender le phénomène mis en jeu. Cependant, il est nécessaire de développer un modèle numérique permettant d'étudier des structures plus représentatives de la réalité pour apporter une réponse globale aux problèmes sédimentaires rencontrés.

L'objectif final étant de simuler les perturbations de l'écoulement engendrées par la présence d'une structure fortement allongée, des artifices permettant de modéliser simplement la structure et de limiter le nombre de mailles doivent être utilisés. Pour cela et compte tenu du comportement de l'écoulement autour de la structure, nous avons choisi de considérer l'ensemble \{table + poches à huîtres\} comme une combinaison de milieux poreux : un pour représenter l'ensemble des poches à huîtres et deux autres pour représenter les deux rangées de pieds de la table (ERRIGUIBLE et al., 2006). L'implantation de ce type de modèle dans un code commercial tel que Ansys Fluent ${ }^{\mathrm{TM}}$ nécessite de fixer uniquement la valeur des paramètres régissant le domaine, à savoir un paramètre de perméabilité $(\alpha)$ et un paramètre de résistance inertielle $\left(C_{2}\right)$ pour chacune des trois directions (YANG et al., 2009), suivant l'équation de Darcy-Forcheimer. Ainsi, on s'affranchit de la définition d'un maillage prenant en compte les multiples particularités géométriques de la structure, ce qui limite par conséquent le nombre de 
mailles nécessaires pour représenter le domaine d'étude. Suite à différents tests et à quelques considérations géométriques (diamètres des pieds suffisamment fins pour ne pas influencer l'écoulement suivant les directions $\mathrm{Oy}$ et $\mathrm{Oz}$, mais dont l'influence cumulée sur la longueur considérée est non négligeable; porosité des poches à huîtres équivalente suivant les directions $\mathrm{Ox}$ et $\mathrm{Oz}$ ), les différents paramètres ont été fixés aux valeurs indiquées dans le tableau 2.

Le nombre de Reynolds caractérisant ce type d'écoulement étant relativement élevé ( $R e=3,6 \times 10^{6}$, pour une vitesse de veine de $0,5 \mathrm{~m} / \mathrm{s}$ et une longueur caractéristique correspondant à une longueur de table de 7,2 m), nous avons retenu le modèle de turbulence SST $\kappa-\omega$ pour la résolution des équations de Navier-Stokes par l'intermédiaire du logiciel Ansys Fluent ${ }^{\mathrm{TM}}$ (MENTER et al., 2003). Un maillage structuré de 200000 mailles (figure 4) avec un raffinement proche des parois est nécessaire pour obtenir la convergence des calculs.

Tableau 2. Valeurs des coefficients de perméabilité et de résistance inertielle retenus pour définir le volume des poches à huîtres et le volume occupé par les pieds de tables.

\begin{tabular}{c||c|c||c|c}
\multicolumn{1}{c||}{} & \multicolumn{2}{c||}{ oysters bags } & \multicolumn{2}{c}{ table legs } \\
\hline Axe & $1 / \alpha$ & $C_{2}$ & $1 / \alpha$ & $C_{2}$ \\
\hline \hline $\mathrm{X}$ & 4 & 10000 & 4 & 0 \\
\hline $\mathrm{Y}$ & 0 & 1000000 & 0 & 0 \\
\hline $\mathrm{Z}$ & 4 & 10000 & 0 & 0
\end{tabular}

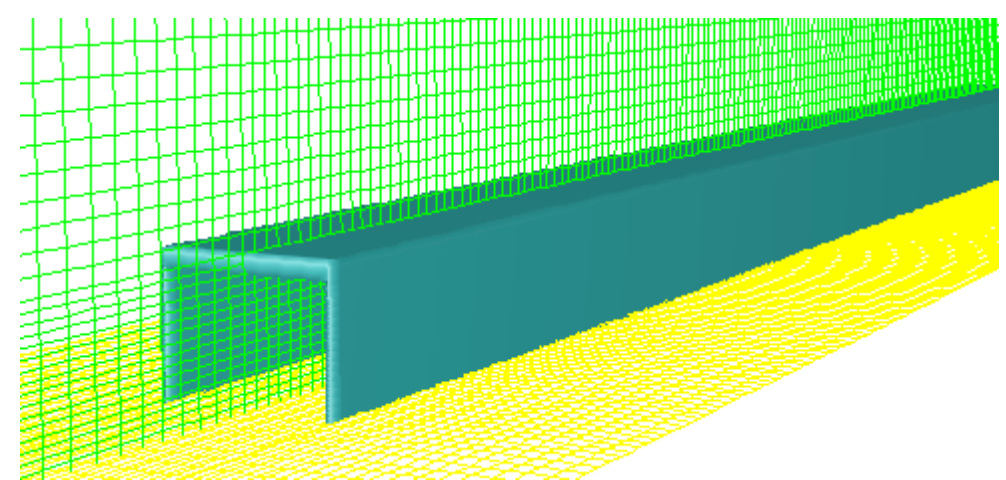

Figure 4. Maillage du domaine, la table étant modélisée à l'aide de milieux poreux.

La comparaison des résultats numériques et expérimentaux montre une bonne concordance. Une erreur moyenne sur l'ensemble du domaine d'étude entre 3 et $10 \%$ est obtenue pour les configurations A, B (dont les résultats sont présentés figure 5) et C. L'erreur maximale peut atteindre ponctuellement pour chacune de ces configurations $20 \%, 25 \%$ et $10 \%$. Ces différences apparaissent uniquement à proximité des poches, cette zone présentant des irrégularités géométriques importantes. La prise en compte des pieds de la table est uniquement justifiée pour la configuration $\mathrm{A}$, où le cumul des effets de sillage entraîne un déficit de vitesse de l'ordre de $40 \%$ sur le bord de la table. 
Des comparaisons sur les niveaux d'énergie cinétique (TKE) obtenus expérimentalement et ceux obtenus numériquement (figure 6) permettent d'asseoir la validation du modèle numérique. Pour la configuration $\mathrm{A}$, une zone de production plus large est obtenue numériquement, mais avec des niveaux comparables avec ceux obtenus expérimentalement : $7 \times 10^{-3} \mathrm{~m}^{2} / \mathrm{s}^{2}$ contre $5 \times 10^{-3} \mathrm{~m}^{2} / \mathrm{s}^{2}$. Pour la configuration $\mathrm{B}$, les zones énergétiques sont comparables, mais avec des niveaux obtenus expérimentalement plus importants : $9 \times 10^{-3} \mathrm{~m}^{2} / \mathrm{s}^{2}$ contre $7 \times 10^{-3} \mathrm{~m}^{2} / \mathrm{s}^{2}$ numériquement.
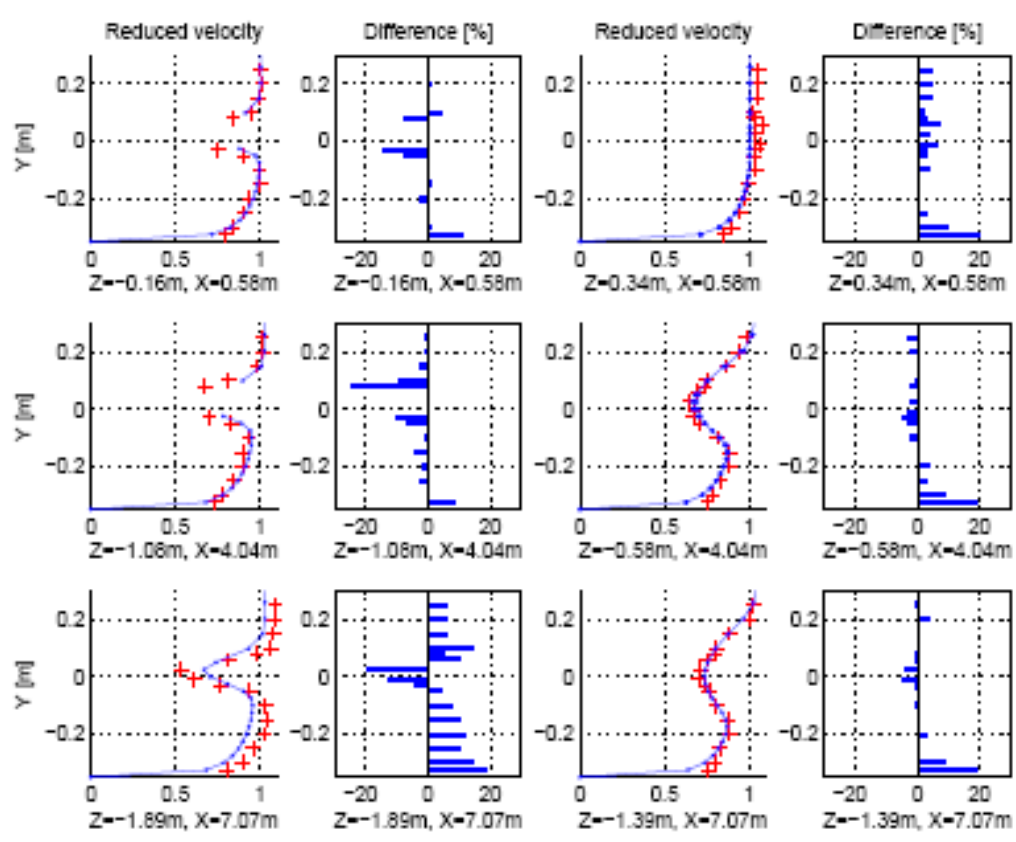

Figure 5. Comparaison entre les résultats numériques et expérimentaux sur différents profils réalisés sur la configuration $B$.

La validation du modèle numérique mis en place à partir des comparaisons présentées précédemment permet d'étendre le modèle pour évaluer les perturbations de l'écoulement engendrées par la présence de tables de grande longueur. Le défaut principal des travaux présentés jusqu'alors porte sur la non concordance de la longueur de la maquette et les longueurs de table rencontrées sur les zones de production. Une possibilité offerte par le modèle numérique développé est de pouvoir étendre la longueur de table considérée par une simple extension suivant la direction voulue.

Après validation de la faisabilité à partir de comparaisons directes entre un maillage étendu (nombre de mailles multiplié par la valeur du facteur d'échelle considéré) et un maillage subissant un facteur d'échelle, nous avons appliqué un facteur d'échelle (facteur 10) dans la direction principale sur le maillage d'une table de 10 m modélisée suivant les choix présentés précédemment. Cet artifice permet d'étudier les perturbations de l'écoulement engendrées par la présence de tables de $100 \mathrm{~m}$ de long et de constater, à partir des champs de vitesses présentés figure 7, que l'écoulement autour 
d'une table d'une telle longueur est relativement différent de celui obtenu jusqu'à présent. Un fort ralentissement de l'écoulement est notamment perceptible sur le dessous de la table, avec l'épaississement de la couche limite initiée sous la table. La couche limite atteint son extension maximale avant les 30 premiers mètres de la structure, pour aboutir à un écoulement stabilisé sur le reste du domaine.

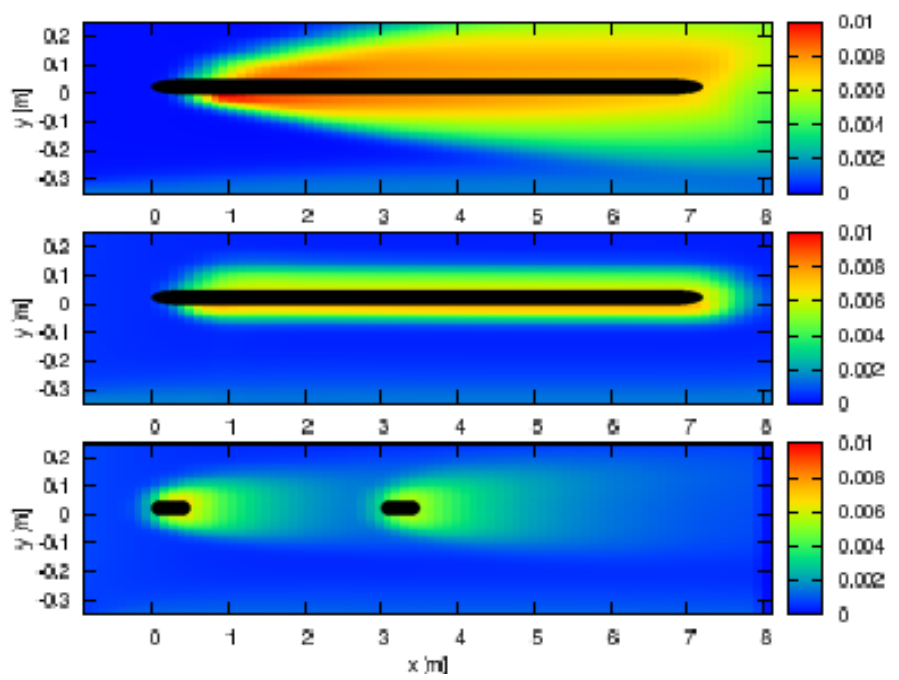

Figure 6. Niveau d'énergie cinétique turbulente $\left(\mathrm{m}^{2} / \mathrm{s}^{2}\right)$ dans le plan de symétrie vertical pour les configurations $A$ (en haut), $B$ (au centre) et $C$ (en bas).

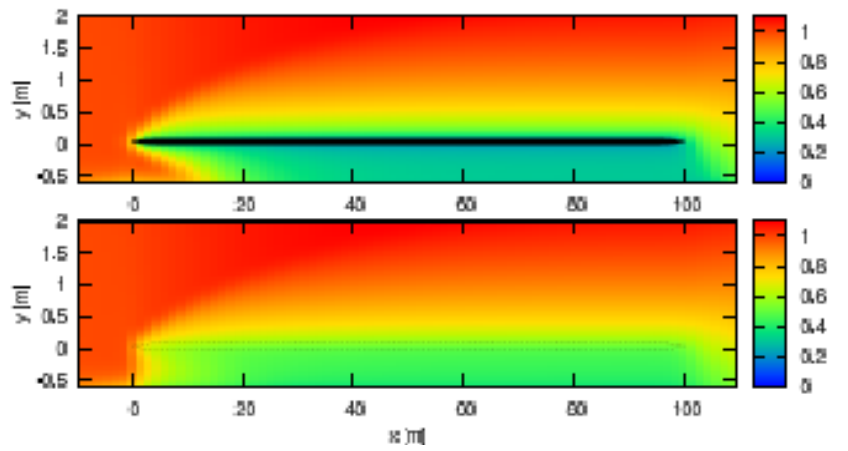

Figure 7. Champ de vitesses axiales normalisé dans le plan de symétrie vertical pour une longueur de table de $100 \mathrm{~m}$, colinéaire à la direction de l'écoulement.

La contrainte de cisaillement près du fond sous une longueur de table de $100 \mathrm{~m}$ présente des valeurs pouvant être assez élevées autour de la structure, alors qu'elle est très faible sous la table (figure $8 \mathrm{a}$ ). Ceci peut expliquer les phénomènes de sédimentation que l'on retrouve sous les tables et au niveau de leur bord de fuite sur certains sites d'exploitation (figure $8 \mathrm{~b}$ en Baie des Veys, France).

La relative simplicité du modèle mis en place et le faible nombre de mailles utilisé pour mailler l'ensemble du domaine permettent d'envisager la poursuite de cette étude pour analyser les effets d'interaction entre plusieurs rangées de tables placées en espace 
proche. A terme il sera possible de déterminer un jeu de paramètres caractéristiques (densité de rugosité, coefficient de friction) permettant de prendre en compte la présence de tables ostréicoles dans des modèles hydrodynamiques côtiers de type MARS (CAYOCCA et al., 2008) pour étudier les effets d'interaction et les perturbations engendrées à l'échelle d'une baie.

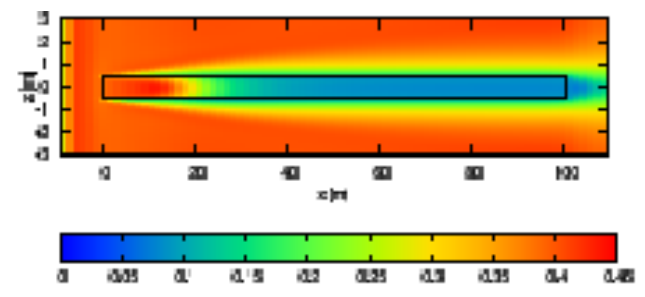

(a)

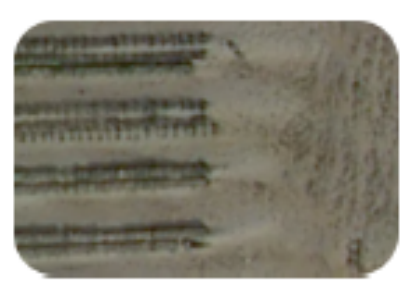

(b)

Figure 8. Evolution de la contrainte de cisaillement près du fond pour une longueur de table de $100 \mathrm{~m}$, colinéaire à la direction de l'écoulement (a) et photographie aérienne à l'extrémité d'un champ de tables des effets de sillage (b).

\section{Conclusions}

Nous avons présenté la méthodologie mise en place pour améliorer la compréhension des perturbations physiques du milieu engendrées par l'implantation de tables ostréicoles et caractériser les modifications de l'écoulement. Ce travail pouvant être difficilement effectué à partir de mesures in situ, nous avons fait le choix de réaliser cette étude à partir du développement d'un modèle numérique permettant de simuler l'écoulement autour de tables ostréicoles. Ce modèle dit de moyenne échelle provient de l'extension d'une modélisation petite échelle, validée à l'aide d'essais expérimentaux.

Ces essais ont notamment permis de caractériser l'impact en champ proche sur l'écoulement. Nous avons mis en évidence des zones préférentielles de dépôts de sédiments dues à l'interaction entre la couche limite se créant sur le dessous de la table et la couche limite de fond. Les contraintes de cisaillement mesurées près du fond ne sont quant à elles pas modifiées par la présence de la structure. Seule la contrainte de cisaillement sur l'ensemble de la colonne d'eau est amplifiée au passage des tables.

Avec l'objectif de généraliser ces travaux, un modèle numérique a été développé à partir du code de CFD Fluent, les tables étant modélisées à l'aide de milieux poreux. La comparaison des résultats numériques et expérimentaux montre une bonne concordance : de 3 à 10\% d'erreur moyenne sur l'ensemble du domaine d'étude suivant la configuration étudiée. Les choix effectués pour représenter la structure permettent d'envisager l'extension du modèle à des échelles plus importantes (plusieurs centaines de mètres) pour une structure seule ou en interaction avec d'autres placées à proximité. Il sera ainsi possible de déterminer un jeux de paramètres caractéristiques (densité de rugosité, coefficient de friction) permettant de prendre en compte la présence de tables ostréicoles dans des modèles hydrodynamiques côtiers de type MARS. 
Des mesures in situ ayant révélées une atténuation forte de la houle (de l'ordre de $20 \%$ pour certaines conditions de houles incidentes) due à la présence de ce type de structure fortement allongée, une extension de cette étude est prévue pour tenter de caractériser finement ce type de phénomène, et ainsi aboutir à une prise en compte complète des effets d'interaction. Des essais expérimentaux sur houle et courant sont ainsi prévus pour valider les futurs développements numériques (méthode SPH ?).

\section{Références bibliographiques}

CAYOCCA F., BASSOULET P., LE HIR P., JESTIN H., CANN P. (2008). Chapter 29 Sedimentary processes in a shellfish farming environment, Mont Saint Michel Bay, France. Proceedings in Marine Science, Elsevier. Vol. 9, pp 431-446. doi:10.1016/S1568-2692(08)80031-9

ERRIGUIBLE A., BERNADA P., COUTURE F., ROQUES M. (2006). Simulation of convective drying of a porous medium with boundary conditions provided by CFD. Chemical Eng. Research and Design, Vol. 84, pp 113-223. doi:10.1205/cherd.05047

HENDRICKS I.E., VAN DUREN L.A., HERMAN P.M.J. (2006). Turbulence levels in a flume compared to the field: Implications for larval settlement studies. Journal of Sea Research, Vol. 55, pp 15-29. doi:10.1016/j.seares.2005.09.005

KERVELLA Y., GERMAIN G., GAURIER B., FACQ J-V., CAYOCCA F., LESUEUR P. (2009). Boundary layer development and shear stresses measurements around oyster tables. AMT'09.

KERVELLA Y. GERMAIN G., GAURIER B., FACQ J-V., CAYOCCA F., LESUEUR P. (2010). Experimental study of the near-field impact of an oyster table on the flow. European Journal of Mechanics / B Fluids. Vol. 29, pp 32-42. doi:10.1016/j.euromechflu.2009.09.002

LE HIR P., CANN P., WAELES B., BASSOULET P. (2008). Chapter 11 Erodibility of natural sediments: experiments on sand/mud mixtures from laboratory and field erosion tests. Proceedings in Marine Science. Elsevier. Vol. 9, pp 137-153.

MENTER F.R., KUNTZ M., LANGTRY R. (2003). Ten years of industrial experience with the sst turbulence model. Turbulence Heat and Mass Transfer, Vol. 4, pp 625-632.

NEPF H., GHISALBERTI M. (2008). Flow and transport in channels with submerger vegetation. Acta Geophysica, Vol. 56, pp 753-777. doi:10.2478/s11600-008-0017-y

SORNIN J.M. (1981). Processus sédimentaires et biodéposition lies à différents modes de conchyliculture. Thèse, Université de Nantes.

VERNEY R., BRUN COTTAN J.C., LAFITE R., DELOFFRE J., TAYLOR J.A. (2006). Tidally-induced shear stress variability above intertadal mudflats in the macrotidal Seine estuary. Estuaries and Coasts, Vol. 29, pp 653-664.

YANG D., YANG Y., COSTA V.A.F. (2009). Numerical simulation of non-darcian flow through a porous medium. Particuology, Vol. 7, pp 193-198. doi:10.1016/j.partic.2009.02.001 